\title{
La relación intersubjetiva en la construcción del conocimiento
}

The Intersubjective Relation in the Construction of Knowledge

Le rapport intersubjectif dans la construction de la connaissance

A relação intersubjetiva na construção do conhecimento

Fecha de recepción: 31 DE JULIO DE 2014 / Fecha de aceptación: 17 DE SEPT. DE 2014 / Fecha de disponibilidad en línea: 30 DE DIC. DE 2014

Encuentre este artículo en http://magisinvestigacioneducacion.javeriana.edu.co/

doi: 10.11144/Javeriana.M7-14.LRIC

Escrito por Lorena Cruz-Garcette

Universidad Nacional de CuYo

Mendoza, Argentina

cruzlorena74@hotmail.com

Graciela Martins de Abreu-Ballester

Universidad Nacional de Cuyo

Mendoza, Argentina

grabreu@yahoo.com.ar

Lía Stella Brandi-Bruna

Universidad Nacional de Cuyo

Mendoza, Argentina

Isbrandi@yahoo.com

\section{Resumen}

El artículo presenta nuestras reflexiones a partir de la reconstrucción de dos experiencias de investigación educativa en Argentina. Nos propusimos analizar las complejas relaciones de fuerza entre los actores involucrados en los procesos de construcción del conocimiento, desde una perspectiva interpretativa y crítica que postula el conocimiento como una construcción intersubjetiva y un espacio de encuentro con el otro, signado por relaciones de poder entre investigadores e investigados. Las conclusiones se formulan en términos de recomendaciones de índole metodológica y ética encaminadas a fortalecer las prácticas de investigación cualitativa en educación.

\section{Palabras clave descriptor}

Conocimiento científico, relaciones interpersonales, investigación pedagógica, investigación participativa.

\section{Transferencia a la práctica}

Si bien el tema de las relaciones de poder en los procesos de construcción del conocimiento no es novedoso, sigue presentándose como un desafío para el investigador cualitativo. En diálogo con nuestras prácticas intentamos aportar algunas reflexiones y recomendaciones para el análisis de las tramas de poder en dos dimensiones: metodológica y ética. En la primera, sugerimos tener en cuenta estrategias propias de la investigación cualitativa: el consentimiento informado, el anonimato, la confidencialidad, la confesión teórica, la confirmación de los participantes, la inclusión de las voces de los actores, la negociación de sentidos y la triangulación. En la dimensión ética, nuestra propuesta es transparentar las relaciones de poder y reflexionar sobre ellas de modo colaborativo.

Para citar este artículo / To cite this article / Pour citer cet article / Para citar este artigo

Cruz-Garcette, L., Martins de Abreu-Ballester, G., \& Brandi-Bruna, L. S. (2014). La relación intersubjetiva en la construcción del conocimiento. magis, Revista Internacional de Investigación en Educación, 7(14), 85-98. 


\section{Key words plus}

Scientific Knowledge, Interpersonal Relationships, Educational Research, Participative Research.

\section{Abstract}

The paper presents our reflections from the reconstruction of two experiences of educational research in Argentina. We analyzed the complex power relations that occurred between the actors involved in the processes of knowledge construction, from an interpretive and critical perspective that posits knowledge as an intersubjective construction, a space of encounter with the "other", marked by power relationships between researchers and those being researched. Conclusions are formulated in terms of recommendations, of methodological and ethical nature, aimed at strengthening the practices of qualitative research in education.

\section{Transference to practice}

While the issue of power relations in the process of knowledge construction is not new, it continues to be a challenge for the qualitative researcher. In dialogue with our practices we intend to contribute with some reflections and recommendations for the analysis of power relations by taking into account two aspects: methodological and ethical dimensions. In relation to the first we suggest to consider the strategies of qualitative research: informed consent, anonymity and confidentiality, theoretical confession, confirmation of participants, including the voices of actors, the negotiation of meanings and triangulation. In relation to the ethical dimension our proposal is to give transparency to the power relations and reflect on them collaboratively.

\section{Mots clés descripteur}

Connaissance scientifique, rapports interpersonnels, recherche pédagogique, recherche participative.

\section{Résumé}

Cet article présente nos réflexions à partir de la reconstruction de deux expériences de recherche éducative en Argentine. Nous avons proposé faire l'analyse de complexes rapports de force qui sont en jeu parmi les acteurs impliqués, dans les processus de construction de la connaissance, depuis la perspective interprétative et critique qui comprend la connaissance en tant qu'une construction intersubjective, un espace de rencontre avec l'autre signé par les rapports de pouvoir entre les chercheurs et les personnes objets de la recherche. Les conclusions ont étés formulées dans les termes de recommandations, de caractère méthodologique et éthique, ces conclusions ont étés menées à renforcer les pratique de la recherche qualitative en éducation.

\section{Transfert à la pratique}

Même si le sujet des rapports de pouvoir dans les processus de construction de la connaissance n'est pas nouveau, il continue à se présenter en tant qu'un défi pour le chercheur qualitatif. En dialogue avec nos pratiques nous essayons d'apporter quelques réflexions et recommandations pour faire de l'analyse des trames de pouvoir, en deux dimensions: la méthodologique et l'éthique. Dans la première on suggère notamment les stratégies propres de la recherche qualitative: le consentement informé, l'anonymat et la confidentialité, l'aveu théorique, la confirmation des participants, l'inclusion de voix des acteurs, la négociation de sens et la triangulation. Dans la dimension éthique notre proposition est celle de rendre transparents les rapports de pouvoir et réfléchir par rapport à ces rapports d'une manière collaborative.
Palavras-chave descritor Conhecimento científico, relações interpessoais, pesquisa pedagógica, pesquisa participativa.

\section{Resumo}

O artigo apresenta nossas reflexões a partir da reconstrução de duas experiências de pesquisa educativa na Argentina. Buscamos analisar as complexas relações de força entre os atores envolvidos nos processos de construção do conhecimento, desde uma perspectiva interpretativa e crítica que postula o conhecimento como uma construção intersubjetiva, um espaço de encontro com o outro marcado por relações de poder entre pesquisadores e pesquisados. As conclusões formulam-se em termos de recomendações de índoles metodológicas e éticas, encaminhadas a fortalecer as práticas de pesquisa qualitativa em educação.

\section{Transferência à prática}

Ainda que o tema das relações de poder nos processos de construção do conhecimento não seja inovador, continua se apresentando como um desafio para o pesquisador qualitativo. Em diálogo com nossas práticas tentamos contribuir com algumas reflexões e recomendações para a análise das tramas de poder, em duas dimensões: metodológica e ética. Na primeira sugerimos considerar de forma especial as estratégias próprias da pesquisa qualitativa: o consentimento informado, o anonimato e a confidencialidade, a confissão teórica, a confirmação dos participantes, a inclusão das vozes dos atores, a negociação de sentidos e a triangulação. Na dimensão ética nossa proposta é tornar claras as relações de poder e refletir sobre elas de modo colaborativo. 


\section{Nuestro posicionamiento}

Acerca de los modos de hacer investigación educativa

Como docentes investigadoras, sabemos que construimos nuestra identidad dentro de un sistema propio de ideas, un marco referencial que, más allá de las opciones teórico-metodológicas, supone un compromiso existencial, un "modo inconmensurable de ver el mundo y de practicar, en él, la ciencia" (Kuhn, 2006, p. 62). Tal compromiso nos obliga a preguntarnos: ¿Qué queremos investigar? ¿Desde qué presupuestos y perspectivas teóricas? ¿Desde qué concepciones de educación y práctica docente? ¿Cómo vamos a investigar? ¿Con quiénes? ¿Para qué?

Estos interrogantes aluden a la cosmovisión filosófica del investigador que determina su opción a favor de ciertos paradigmas o teorías, a los que adopta, adapta, critica o crea de acuerdo o en contraposición con otros pensadores, predecesores o contemporáneos. La manera en que respondemos a estos interrogantes y resolvemos los problemas que nos presenta el proceso de investigación nos posiciona de distinta forma respecto a los modos de hacer investigación.

Ubicadas en la mesa del debate, hemos reconocido tres modos legítimos de hacer ciencia de lo social, no contradictorios, pero sí diferentes. Nuestros referentes están representados por la teoría de los intereses constitutivos de los saberes de Habermas (1982), la construcción teórica de Carr y Kemmis (1988) y las tesis de Vasilachis (1992) acerca de la coexistencia de tres paradigmas en la Sociología: positivista, interpretativo y sociocrítico, que responden a los intereses técnico, práctico y emancipador, respectivamente.

Como equipo de investigación, optamos por la perspectiva latinoamericana de investigación educativa que se inscriben en la Sociología interpretativa y crítica y sitúan la problemática educativa en el marco de las prácticas cotidianas en la escuela, sin desconocer el entramado histórico y cultural dentro del cual dichas prácticas adquieren sentido y el modo en que se distribuye el poder simbólico en la cultura escolar.

\section{Acerca de la relación intersubjetiva en la construcción del conocimiento}

Entendemos que la construcción del conocimiento deviene de la interacción entre sujetos, del encuentro entre un sujeto cognoscente y un sujeto conocido (Vasilachis, 2009) en un contexto sociohistórico particular. Por lo tanto, el conocimiento construido estará delineado por las características de esta interacción (Enosh \& Ben-Ari, 2009). Este planteamiento pone en cuestión el lugar del docente investigador.

En la perspectiva tradicional y positivista de la investigación, el investigador es quien define esa interacción desde la supuesta neutralidad y objetividad que sustenta como principio. Prima aquí la epistemología del sujeto cognoscente. El otro, el investigado, como partícipe en la construcción del conocimiento, es acallado, aislado y silenciado; aparece como un objeto (Vasilachis, 2009). La relación es desigual porque es el investigador quien posee y ejerce el poder sin cuestionarse ni explicitar nada, porque tiene la autoridad que le brindan los principios ontoepistemológicos del paradigma positivista. Se revela una clara visión estática del poder: uno la ejerce sobre el otro.

En cambio, tanto en el paradigma interpretativo como en el paradigma sociocrítico, el sujeto cognoscente se asume como un componente más en la interacción junto con el investigado. En esta interacción no solo se ponen en juego los significados del investigado, pues los significados del investigador también están siendo desafiados; la influencia es mutua
Descripción del artículo | Article description | Description de l'article | Artigo descrição

Este artículo de reflexión se deriva de dos investigaciones "El fracaso escolar y el lugar del conocimiento en la escuela. Un estudio cualitativo en dos instituciones de nivel medio, y la transposición del conocimiento oficial en circuitos escolares diferenciados. Un estudio etnográfico en una escuela urbano-marginal, de nivel medio" y "Las prácticas docentes en espacios curriculares de las carreras de Ciencias de la Educación y Formación Docente de la Facultad de Filosofía y Letras. Un estudio interpretativo desde los actores". 
(Cornejo \& Salas, 2012). El investigador se reconoce como portador de un marco de referencia ontoepistemológico y un cúmulo de percepciones, miradas y prejuicios que debe vigilar a lo largo de todo el proceso de indagación. La interacción que desencadena el encuentro implica una relación de poder (Foucault, 1999) entre el sujeto investigador y el sujeto investigado (Lim, 2012). El investigador interpretativo y el sociocrítico admiten que el sujeto no está separado del objeto y que en la investigación del objeto quedan siempre huellas del sujeto. En el paradigma sociocrítico, el investigador es también el sujeto investigado en una relación orientada a descubrir qué condiciones objetivas y subjetivas limitan los discursos y las prácticas sociales y cómo podrían transformarse unas y otras.

Algunos autores (Cornejo, Besoaín \& Mendoza, 2011; Vasilachis, 2009; Wiesenfeld, 2000) destacan la importancia de generar una relación simétrica, horizontal y de cooperación entre investigadores e investigados en la construcción del conocimiento. Aquí se está reconociendo al sujeto conocido como un otro activo, poseedor de un saber que se interrelaciona con el saber del sujeto cognoscente; ambos aparecen como sujetos activos en este proceso de construcción o co-construcción (Karnieli-Miller, Stier \& Pessach, 2009; Sisto, 2008). Esta mirada sugiere que desde algunas perspectivas epistemológicas es posible y deseable generar puentes entre la perspectiva del investigador y la perspectiva del actor (Wiesenfeld, 2000). En este encuentro, el poder del investigador y la relación asimétrica tienden a achicarse $y$, en algunos casos, a desaparecer, como en la investigación-acción participante, propuesta metodológica con una fuerte racionalidad emancipadora y transformadora de las desigualdades sociales.

Sin negar el valor de la relación horizontal en el proceso de construcción del conocimiento y de acuerdo con Foucault (1999), entendemos que la interrelación entre investigadores e investigados no escapa a las relaciones de fuerza que se desencadenan en cualquier tejido social. Desde esta concepción, consideramos que la equidad y horizontalidad entre los participantes de la investigación es irreal (Ben-Ari \& Enosh, 2013; Enosh \& BenAri, 2009; Vorraber, 1997), tanto en los estudios interpretativos como en la investigación-acción. Si bien son dos sujetos activos que se encuentran en un espacio concreto, cada uno de ellos tiene propósitos y tareas distintas. Las diferentes posiciones que ocupan en el campo social se traducen en relaciones de fuerza que, lejos de ser estáticas y unilaterales, son dinámicas y complejas (Lim, 2012).

El tipo de investigación, el contexto sociohistórico y cultural, las características de los participantes y los diversos significados y las acciones que construyen configuran a lo largo del proceso de investigación una trama de relaciones que inclinan la balanza hacia un lado u otro según las visiones de mundo, tradiciones y trayectorias de los actores involucrados y los momentos o las instancias del proceso de investigación. Otro rasgo que interviene en esta relación es la fuerza de lo burocrático, que puede manifestarse en el poder del informante de resistirse a brindar información o el de la comunidad científica que impone determinados criterios de credibilidad.

Desde nuestra experiencia en investigación educativa, las relaciones de poder entre el investigador y los participantes se construyen en un movimiento constante, asumen distintas formas y se transforman según los significados, las acciones que se ponen en juego y las condiciones en que se realizan. Sin embargo, advertimos que en esta relación de poder entre investigador e investigados, en la que ambos quedan expuestos en sus subjetividades, es el sujeto cognoscente quien asume mayor 
responsabilidad. Es por ello que, como investigadores cualitativos, vivimos esta práctica de investigación, nada sencilla de transitar, con un fuerte compromiso ético.

Hemos asumido que tomar conciencia del interjuego de fuerzas en investigación descomprime y neutraliza la creencia en un poder arbitrario, aséptico y objetivo que actúa desde lo oculto. Con base en nuestra práctica, reconocemos como una estrategia pertinente transparentar y compartir con los actores la existencia de tales relaciones de poder. Esto exige al investigador desarrollar acciones de reflexión y de vigilancia durante todo el proceso de investigación y de modo colaborativo (Ben-Ari \& Enosh, 2010; Breuer, 2003; Declan, 2014; Sisto, 2008).

En este marco, como investigadoras cualitativas, nos preguntamos: ¿Cómo se construyen las tramas del poder entre investigadores e investigados? ¿Quiénes y cómo intervienen en la construcción del conocimiento? ¿Quiénes tienen la palabra? ¿Qué voces se escuchan y qué voces se acallan? ¿Quiénes construyen los datos? ¿Qué interpretación es la que cuenta? ¿Quién autoriza la publicación de los resultados?

Presentamos algunas reflexiones sobre los modos como se distribuye el poder simbólico en los procesos de investigación educativa, surgidas de dos estudios que inscribimos en el paradigma interpretativo y en el sociocrítico, respectivamente, con predominio del enfoque etnográfico en el primero y de la investigación-acción participante en el segundo.

\section{Las relaciones intersubjetivas en dos experiencias de investigación cualitativa en Argentina}

Un estudio cualitativo con enfoque etnográfico sobre el fracaso escolar y el lugar del conocimiento en escuelas de nivel medio pertenecientes a circuitos escolares diferenciados

\section{- La investigación}

El estudio se realizó en San Juan (Argentina), en el Instituto de Investigación en Ciencias de la Educación (Idice), dependiente de la Universidad Nacional de San Juan, entre 1994 y 1998. Sabíamos que la distribución de conocimientos no se realiza de la misma manera en segmentos diferenciados del sistema educativo argentino (Braslavsky, 1985) y, por lo tanto, valía la pena estudiar para comprender este fenómeno en contextos socioeducativos diferentes.

La mirada crítica de la problemática del fracaso escolar — no tanto en relación con el acceso y la permanencia en el sistema, sino más bien con los procesos de distribución y apropiación de conocimientos de relevancia social - fue clave en la construcción del objeto de estudio. En la primera etapa estudiamos los procesos de transposición del conocimiento escolar y en la segunda, las mediaciones institucionales que operan entre el saber prescripto en la propuesta oficial y el saber efectivamente enseñado, en una escuela de sectores populares.

Como referente empírico seleccionamos dos instituciones escolares de nivel medio: la escuela $A$, dependiente de la Universidad Nacional de San Juan y la escuela B, dependiente del Ministerio de Educación de la Provincia. Definimos como unidades de análisis las clases de primer año, cuarta división de Lengua e Historia en ambas escuelas y como fuentes documentales utilizamos el currículo oficial, los planes y programas de estudio, los documentos que dan cuenta de los orígenes y el devenir institucional, los textos escolares y los cuadernos de los alumnos y las matrices de datos de matrícula y rendimiento escolar. 
Las preguntas iniciales fueron: ¿Qué conocimiento se distribuye en la escuela media? ¿Cómo se distribuye y circula el conocimiento en segmentos escolares diferenciados? ¿En qué condiciones se realizan estos procesos? ¿Cuál es la relación con el fracaso y el éxito escolar? Se orientaron a precisar el lugar del conocimiento en la escuela y nos llevaron a centrar nuestra atención en el contenido y la interacción verbal como aspectos privilegiados de la transposición didáctica (Chevallard, 1991), bajo la creencia de que ambos intervienen en el pasaje del conocimiento erudito al conocimiento enseñado. De esta manera, el modelo de análisis propuesto, construido en diálogo con los significados locales, está enfocado en la textualización del conocimiento como eje de análisis en su doble vertiente: la lógica del contenido y la lógica de la interacción verbal.

En la segunda etapa, la complejidad del fenómeno estudiado nos condujo a vincular las observaciones realizadas con el análisis de los estilos y dinámicas institucionales. Las condiciones de trabajo y las prioridades que estipulamos para esta segunda instancia nos llevaron a profundizar el estudio solo en la escuela B. Esta escuela se conoce en el medio con el apelativo de "El arca de Noé", que se enuncia de modo peyorativo para significar a la población escolar en términos de toda especie de animales en búsqueda de salvación.

Avanzamos en el estudio de algunos componentes constitutivos de la cultura y el estilo institucional que construye la escuela B: el espacio material y simbólico, la novela institucional y la ideología institucional que analizamos en su relación con los modos particulares de transposición del conocimiento en la escuela.

Nuestros análisis muestran que el contenido guarda mayor relación con el conocimiento erudito en la escuela A que en la escuela B. En esta última, el sujeto pedagógico, percibido en términos de sujeto carenciado, genera bajas expectativas y lógicas de transposición que tienden a excluir del conocimiento a una población que se incluye solo en términos de disciplina y formación moral.

La escuela B se debate entre cumplir con "la misión salvadora" legada por el mandato fundacional o resignarse ante la idea de la imposibilidad de intervenir desde una cierta "actitud fatalista" que condiciona todos los aspectos de la vida institucional.

- Las relaciones intersubjetivas en el proceso de investigación

La metodología adoptada supuso riesgos y desafíos que, en algunos casos, no pudimos resolver. Nuestras percepciones, no siempre formadas para mirar y escuchar a los otros y entender qué hacen y piensan, en lugar de proyectar lo que "deberían" hacer y pensar nos sometieron a una fuerte tensión entre nuestras perspectivas y las que sostienen los participantes.

Si bien se produjeron encuentros y desencuentros con los actores institucionales durante la investigación, seleccionamos dos momentos clave que dan cuenta de la relación de fuerzas que entablamos: uno de ellos, vinculado con el acceso al campo y el otro, con los nombres de la investigación.

Como estrategia de acceso, buscamos legitimación formal en los cargos jerárquicos más altos del sistema educativo provincial. En la base de las decisiones tomadas había una clara intención de asegurarnos el acceso a las escuelas, que tiene su historia en una experiencia anterior en la escuela B. Esta estrategia generó una tensión inicial en la relación con el personal directivo y docente. En la primera entrevista, la directora nos dijo: "La secretaria de educación me manda los alumnos fracasados y después me las manda a ustedes para que estudien el fracaso escolar" (comunicación 
personal, 6 de marzo de 1995), con lo que expresó su posición respecto a la autoridad escolar, al reconocimiento de la existencia de alumnos fracasados en la escuela y su reticencia hacia nuestro trabajo.

Josefa Berenguer, integrante del equipo de investigación, analizó "la entrada a escena", con base en la metáfora "la vida social es una escena", de Goffman (1974). Según la investigadora, para recomponer la relación, nuestros actos se caracterizaron por dos actitudes: la deferencia (avales institucionales, avisos y concertaciones previas de visitas y entrevistas) y la reparación (presentaciones personales, profesionales y del proyecto en reiteradas oportunidades). Entendimos que el "acceso" a la escuela no se asegura de una vez para siempre sino que se negocia todo el tiempo.

En cuanto a los nombres del proyecto, el título inicial era: "Pobres de cuna' y 'desertores natos'. Un estudio cualitativo en una escuela de sectores populares". Nuestra intención fue utilizar dos categorías locales que nos habían sorprendido por su carga estigmatizante durante las actividades preliminares del trabajo de campo. Los docentes afirman que los alumnos de la escuela se dividen en los que vienen de las villas ${ }^{1}$ - "los pobres de cuna" - y los que han fracasado en otras escuelas - "los desertores natos"- . Fue un desencuentro significativo entre nuestras perspectivas y las de los actores, momento que Willis (1980) considera de máxima alteración para los investigadores, cuyos propios significados están siendo profundamente confrontados. La disidencia se manifestó también con nuestros pares que, en diversos eventos de comunicación científica, nos cuestionaron el título de la investigación. Aunque ambas categorías tenían comillas, muchos no advirtieron la connotación crítica que queríamos destacar. Si bien fue aprobado por las autoridades pertinentes, tomamos la decisión de cambiar el título del proyecto.

La tensión reapareció en el momento de la publicación de los resultados, esta vez por nuestra intención de titular el libro El arca de Noé y la negativa de algunos docentes. Desde nuestra perspectiva, la metáfora anticipaba el contenido de la investigación y denunciaba, al mismo tiempo, el estigma social que reciben alumnos y docentes de escuelas de sectores populares. Advertíamos que el apelativo es vivido como una afrenta por la comunidad educativa porque tal vez "desafía la intención del orden instituido de suprimir todas las diferencias a través de un discurso que afirme la homogeneidad del dominio social" (Giroux \& Simon, 1998, p. 190). Sin embargo, es la sabiduría popular la que pone al descubierto la falacia de igualdad que sostienen las escuelas de sectores populares.

1 En Argentina se denominan "villas" a los asentamientos de viviendas precarias con carencia de infraestructura y pobreza urbana.
Analizar el entramado histórico, político y cultural dentro del cual adquieren sentido dichas prácticas nos permitió comprender que, así como la escuela se atribuye la tarea de salvación, el sistema le asigna la función particular de recibir a los que no tienen lugar, de modo que ocupa un lugar residual dentro de la estructura del sistema. Los docentes están tan limitados como los alumnos por la adversidad de las condiciones en las que trabajan. La falta de reconocimiento oficial de las condiciones en las que se desarrolla la tarea en la escuela no puede obviarse a la hora de explicar la lógica institucional descrita.

Esta trama que logramos reconstruir más la negativa de algunos docentes de la escuela B para publicar los resultados con el nombre de El arca de Noé, motivada por el deseo de resguardar el prestigio social de la institución, nos llevó a renunciar a nuestra intención inicial.

Tanto la entrada al campo como los desencuentros a la hora de nombrar la investigación dan cuenta de la compleja trama de relaciones de poder que construimos investigadores e investigados. Allí se confrontaron significados y marcos referenciales diversos, el origen histórico de la situación, las tradiciones pedagógicas subyacentes y el sustrato ideológico sobre el que se basan. Surgieron fuerzas de distinto tipo en cada momento de la investigación, los actores institucionales resistieron nuestra presencia en dos oportunidades y terminaron imponiendo su postura a la hora de la publicación de los resultados. No obstante, en el análisis y la interpretación primaron nuestros marcos referenciales. Nosotras miramos la escena y recogimos la información que examinamos desde las perspectivas que habíamos transparentado en nuestra "confesión teórica" (Willis, 1984).

Una particular fuente de poder fue nuestro saber pedagógico, que nos posicionaba en mejores condiciones para escribir, argumentar y contraargumentar sobre las prácticas cotidianas en la escuela. También es posible que nuestra pertenencia a la universidad, en calidad de especialistas en Educación, hubiera operado como fuente de conflicto. En nuestro país existe una tradicional pugna entre pedagogos y docentes por el control de la enseñanza, que profundiza la escisión teoría-práctica en el campo de la Educación.

En esta relación de fuerzas predominaron algunos significados: por nuestra parte, tuvo preponderancia el malestar que nos causaba presenciar distintos modos locales de producir desigualdad y estigmatización social; de allí nuestra intención de develarlos. Al mismo tiempo que nos interesaba generar conocimiento en forma de entendimiento interpretativo, entendíamos que nuestra interpretación reveladora de prácticas estigmatizantes en la escuela $B$ serviría para que los docentes involucrados pudieran repensar sus 
prácticas. En los actores institucionales prevaleció el imperativo de preservar la imagen de la escuela ante la comunidad educativa.

Nuestro dilema ético se planteó, entonces, entre las exigencias del paradigma emancipador, según el cual debíamos sacar a la luz las construcciones discursivas y las prácticas cotidianas en la escuela, como una forma de producir transformaciones curriculares orientadas hacia formas más justas y liberadoras y, por otro lado, la necesidad de resguardar el prestigio de la institución y proteger la privacidad y los intereses de los actores involucrados.

Aunque desde la teoría se insiste en la necesidad de no abrir juicios en investigación cualitativa y se pone el acento en las maneras de comprender el mundo de los actores y en el respeto al valor de sus conocimientos, nuestra experiencia nos dice que la lectura interpretativa en Ciencias Sociales siempre conlleva evaluación y juicio. Coincidimos con el análisis de Schuster y Pecheny (2002) sobre la problemática de la comprensión en Ciencias Sociales según Habermas:

Para explicar en Ciencias Sociales es necesario comprender el sentido de las acciones, para comprender dicho sentido es necesario comprender las pretensiones de validez inherentes a las acciones; dichas pretensiones se apoyan argumentativamente en razones que son susceptibles de juicio, por ende, explicar en Ciencias Sociales implica juzgar [...] este enfoque plantea que no sólo no hay que neutralizar los valores, sino que para aspirar a la objetividad en las Ciencias Sociales, es necesario juzgar, tomar partido (p. 240).

Eso fue lo que hicimos: decidimos publicar los resultados de nuestras investigaciones. En una suerte de negociación con los docentes de la escuela B, cambiamos el título del libro y nos ocupamos de resguardar la identidad de la escuela y de sus actores institucionales.

Según Carr y Kemmis (1988), entendemos que la tarea realizada aporta un conocimiento valioso acerca de las condiciones institucionales que pueden estar distorsionando "la autorreflexión y el autoentendimiento, para posibilitar [...] la autoemancipación de los seres humanos" (p. 149). No obstante, como sugieren los autores, hemos comprendido que esa emancipación debe ser "mediante sus propios entendimientos y actos y la búsqueda de la libertad y autonomía personal" (p. 149).

Esta resignificación epistemológica ha constituido para nosotras un valioso avance en la configuración de nuestros marcos referenciales, que transitan desde un interés práctico e interpretativo hacia una racionalidad sociocrítica y emancipadora y su correlato metodológico: la investigación-acción participante.

Una práctica de investigación-acción participante

de profesores universitarios constituidos

en comunidad autocritica

\section{- La investigación}

El estudio se desarrolló en Mendoza (Argentina), en el Instituto de Ciencias de la Educación (ICE) de la Universidad Nacional de Cuyo, entre 2009 y 2011. Se inscribe en la línea de práctica docente, en la que trabajamos desde 2003. Nos propusimos reflexionar para comprender y transformar nuestras prácticas en asignaturas de formación docente en la carrera de Ciencias de la Educación y en los profesorados de la Facultad de Filosofía y Letras de la UNCuyo. Optamos por un enfoque interpretativo y crítico de investigación-acción, que exige la participación del docente investigador en la acción social que se estudia. Ello implica un proceso colaborativo de autorreflexión que se materializa en comunidades autocríticas de practicantes comprometidos en mejorar la educación.

El propósito fue mirarnos a nosotras mismas, decodificar el discurso que producimos, los sentidos de nuestras prácticas, los modos de desarrollar la enseñanza y el vínculo con el conocimiento, la tarea y los alumnos.

Las preguntas iniciales que orientaron las distintas instancias del trabajo de campo y el análisis de la información fueron: ¿Qué transmitimos con nuestro modo de ser docente en nuestras clases? ¿Cuál es la posición de cada docente ante los problemas de la práctica docente? ¿Cuáles son los aspectos más conflictivos? ¿Qué bases teóricas y valores socioeducativos sustentan nuestras prácticas? ¿Cómo perciben los alumnos la dimensión ética y política de nuestras prácticas?

Seleccionamos como unidades de análisis: a) Didáctica y Currículum, tercer año, común a los profesorados de Filosofía, Geografía, Historia, Letras y Lenguas Extranjeras; b) Didáctica I y Trabajo de Campo, y c) Metodología de la Investigación II, ambas de tercer año de la carrera de Ciencias de la Educación.

Recogimos la información mediante observaciones y notas de campo de clases y reuniones de equipos de cátedra y de investigación que giraron en torno a nuestras prácticas: lectura de marcos teóricos, análisis de los materiales empíricos recogidos, confrontación de marcos de referencias, puntos de vista y modos de entender la docencia universitaria.

Hicimos el análisis de la información con el método comparativo constante según Sirvent (1998) y la propuesta de análisis de Berteley-Busquets (2000). De este modo, avanzamos en la construcción de una des- 
cripción analítica que resultó de triangular las perspectivas de los actores y la información proveniente de las reuniones de equipo y las clases de algunas asignaturas, en especial las de Didáctica I y Trabajo de Campo, de Ciencias de la Educación.

Así, pudimos examinar nuestras prácticas como expresión de perspectivas teóricas, intenciones políticas y visiones de mundo, con el objeto de construir un conocimiento significativo desde un punto de vista institucional, histórico y social. Logramos generar mecanismos de comunicación y participación en las cátedras y nos propusimos continuar y fortalecer las instancias de análisis y reflexión en el equipo, con la intención de proponer nuevas estrategias para la acción pedagógica.

\section{- Las relaciones intersubjetivas en el proceso de investigación}

El proceso de construcción del conocimiento no fue sencillo ni lineal. El trayecto recorrido nos revela un proceso de aprendizaje profundo e intenso como comunidad autocrítica, en donde hubo momentos de encuentro, comprensión crítica, respeto, compromiso y responsabilidad, pero también instancias de tensión, perspectivas encontradas, confrontación, alianzas, mediación y negociación. Como integrantes de la comunidad autocrítica, sabíamos que nos exponíamos a la mirada de nuestros colegas y al desafío que conlleva. Asumimos que esta tarea implica un compromiso diario con nosotras mismas como equipo de investigación, con nuestros estudiantes y con la institución dentro de la cual estamos insertas; podemos decir que hemos abierto espacios de autocrítica intra e intercátedras.

En el proceso de investigación se nos presentaron diversas dificultades que dan cuenta de las relaciones de fuerzas en el equipo, algunas vinculadas con aspectos organizativos de la tarea y otras con el significado mismo de la investigación acción participante, en especial con el "para qué" de la docencia y la investigación.

Las dificultades organizativas se nos presentaron en términos de tensión entre los miembros del equipo y se manifestaron en limitaciones referentes a acordar los tiempos de reuniones y sostener un proceso de análisis y reflexión continua; hacer las lecturas sugeridas por cada integrante; articular marcos teóricos y metodológicos de referencia; propiciar un clima en el que todas las participantes pudiéramos expresar nuestros puntos de vista; poner en tensión, mediar y negociar perspectivas teóricas en distintos momentos del proceso; argumentar y contraargumentar con la debida fundamentación y producir en forma escrita nuestras reflexiones y avances.

En aras de preservar la continuidad del proyecto, en el último tramo las tareas organizacionales recayeron en quienes habíamos impulsado la investigación acción. Esto provocó una distribución diferencial de poder en la toma de decisiones.

Respecto a los sentidos de la docencia y la investigación-acción participante, la metodología adoptada nos permitió entender que nos movemos entre dos concepciones ontoepistemológicas —una de ellas prácticodeliberativa y la otra crítico-emancipadora- que se expresan con claridad en nuestros discursos y nuestras prácticas a lo largo de todo el proceso de investigación.

El debate y la confrontación entre ambas perspectivas se centraron en las docentes investigadoras de mayor trayectoria, profesoras titulares y adjuntas, que tomamos posición al momento de preguntarnos el "para qué" de nuestras prácticas. Es esclarecedor el planteamiento de PérezGómez (1993), cuando expresa que dentro de esta amplia perspectiva de reflexión sobre la práctica: 
[...] es necesario considerar desde aquellos autores que se manifiestan abiertamente defensores de trabajar y desarrollar en la escuela y en el aula una propuesta ética concreta de justicia, igualdad y emancipación social en los procesos de enseñanza y en los programas de formación de los profesores (Giroux, Smith, Zeichner, Apple, Kemmis) hasta aquellos otros que desde posiciones más liberales defienden la coherencia ética entre los principios, intencionalidades y procedimientos educativos democráticos, sin especificar de antemano un modelo concreto de sociedad (Stenhouse, Elliot, McDonald) (p. 422).

Sobre estas diferencias discutimos con amplitud quienes postulábamos una concepción de la enseñanza como ruptura epistemológica orientada a la transformación sociocultural y, de otra parte, quienes proclamaban una concepción de enseñanza como acompañamiento de procesos interpretativos orientados a comprender los sentidos de la realidad social y educativa, $\sin$ la búsqueda de transformaciones radicales.

En esta confrontación intervinieron varias fuerzas que se manifestaron en relaciones de jerarquía: los cargos docentes, la formación disciplinar y la trayectoria en investigación educativa. En esta trama se evidenciaban alianzas, lealtades, saberes, rasgos de personalidad, diferencias ideológicas y temor a exponerlas, producto de una cultura institucional y social con resabios de autoritarismo. No obstante, creemos que esta experiencia no fue un logro menor, dado que llevábamos diez años trabajando juntas y pocas veces nos habíamos concedido los tiempos necesarios para abrir el debate en torno a estas cuestiones.

A modo de reflexiones finales y para retomar los objetivos del proyecto, como cierre de la primera etapa de la investigación podemos decir que en gran parte logramos constituir una comunidad autocrítica de investigación, describir e interpretar la lógica de nuestros discursos y nuestras prácticas docentes en el contexto socioinstitucional en el que se producen y avanzar en la configuración de una descripción analítica que articula prácticas, sentidos y significados que sustentan docentes y alumnos.

En este proceso de investigación-acción en el que pretendíamos avanzar hacia relaciones de fuerza más equilibradas, se mostraron tensiones resultantes de la contextualización institucional que no siempre tendieron al mentado equilibrio, debido a la ausencia de tiempos y espacios institucionales destinados al debate y a la construcción colaborativa, factores necesarios para desarrollar este tipo de investigaciones. La ausencia de estas condiciones institucionales agravan las siempre presentes relaciones de poder.

A pesar de las dificultades, avanzamos en saber dónde y en qué condiciones estamos trabajando, en comprender que es difícil la confrontación y el debate, en entender que las fuerzas y las tensiones están muy presentes en una institución que se organiza en base a jerarquías, en generar un lazo de confianza entre algunos miembros del equipo que se traduce en nuevos proyectos de investigación, en revisar nuestras propuestas de enseñanza y en la elaboración de propuestas alternativas para la acción pedagógica.

Tal vez los avances más significativos como docentes investigadoras radiquen, por un lado, en haber aceptado el desafío de convertirnos en comunidad autocrítica de investigación y sostener la propuesta y, por el otro, en avanzar en la construcción de un conocimiento que da cuenta de prácticas de investigación-acción en ámbitos universitarios y en un contexto sociohistórico latinoamericano con sus particularidades y obstáculos y que nos ha permitido resignificar y llevar adelante esta propuesta metodológica en las condiciones en las que nuestras realidades lo permiten. 
Para cerrar, traemos las apreciaciones de una reconocida docente investigadora argentina, Ana María Zoppi, que tuvo la gentileza de leer y evaluar nuestro trabajo. Al respecto dice:

A mi juicio, el trabajo es muy interesante por su carácter pionero; no hay todavía en el país investigaciones en las cuales se cumpla sistemáticamente el propósito de analizar la propia práctica. En general, en la bibliografía disponible se registran y ponen en valor buenas prácticas, pero no se toma como objeto la totalidad de la acción en un proyecto de enseñanza y de investigación-acción. Permite documentar cuestiones inevitables en la trama sociológica de la educación, tales como: la presencia activa y diversa de personas que juegan en el mismo escenario; la ineludible marca de sus trayectorias, generando las posibilidades y los límites de los encuentros interpersonales en el equipo; la posibilidad de reconocer las diferencias ideológicas, políticas, científicas, para advertir como éstas siguen estando en la base de distintas posiciones, sin que los acuerdos discursivos puedan anularlas; la señal clara, ejercida y valorada por los mismos protagonistas, de que la deliberación es la condición y la vía para un trabajo colectivo que se pretende, cuando menos, democrático. La apertura a futuro que puede significar para los participantes que deseen involucrarse en procesos reales de cambio y comprensión de las ideas alternativas de algunos compañeros admite la explicitación de que educar no es orientar hacia el vacío. El no cuestionamiento de las estructuras de poder y de discriminación vigentes, plantea un horizonte pedagógico en el que la modernización técnica y el control para preservar los valores tradicionales se sobre imponen. [...] se podrían generar expectativas de organización de la acción desde algún ideal colectivo, en el que se canalice la capacidad política y el compromiso social de los actores. A futuro también nos queda el desafío de encontrar, ojalá con sabiduría y sin fundamentalismos, la posibilidad de sostener la democracia, con su correlato de diversidad y la lucha concreta por la justicia, la igualdad y la emancipación, como necesidades que deben ser atendidas en su conflictiva coexistencia.

\section{Reflexiones finales}

A partir de nuestra experiencia como docentes investigadoras, concebimos el proceso de investigación como ámbito de conflictos entre los actores involucrados, arena de lucha (Ben-Ari \& Enosh, 2013) en la que se libra y ejerce el poder, una práctica social orientada a fines $y$, por lo tanto, saturada de opciones éticas y políticas. Podemos tener más o menos conciencia de tal complejidad, desde creer que son prácticas neutrales, inocentes y transparentes hasta tener plena conciencia de qué estamos haciendo y con qué fin y preguntarnos, por ejemplo, quiénes se benefician con nuestras investigaciones.

En este proceso se configuran tramas de sentidos y acciones en las que intervienen las fuerzas subjetivas de investigadores e investigados, las condiciones institucionales y el contexto sociohistórico y cultural más amplio en los que acontece la construcción del conocimiento. En este movimiento constante de fuerzas, todos, investigadores e investigados, estamos expuestos con mayor o menor intensidad según el momento de la investigación, las decisiones que tomamos o el lugar que ocupamos en el campo. Aquí se exponen el estatus profesional, las perspectivas teóricas y epistemológicas, las trayectorias de vida, los saberes cotidianos y las visiones de mundo de los actores involucrados. 
Comprender las relaciones de poder como constitutivas del tejido social y tener la vivencia del carácter conflictivo y contradictorio de estas relaciones intersubjetivas en la investigación (Lim, 2012) nos posiciona en un lugar de mayor responsabilidad frente al sujeto investigado. Desde un punto de vista metodológico, nos exige vigilar el proceso mediante estrategias propias de la investigación cualitativa: el consentimiento informado, el anonimato, la confidencialidad, la confesión teórica, la confirmación de los participantes, la inclusión de las voces de los actores, la negociación de sentidos y la triangulación.

Desde un punto de vista ético, la tensión se manifiesta en términos de dilemas difíciles de resolver, que comprometen a investigadores e investigados. La acción ética, en este caso, estriba en transparentar las relaciones de poder y reflexionar sobre ellas de modo colaborativo, pues solo con la sinceridad de todos los actores involucrados podría restablecerse un equilibrio entre fuerzas contrapuestas, pese a saber que el balance justo es inalcanzable.

Se trata de exponer y abrir el juego de la participación. Hemos aprendido que comprender otros códigos requiere de un intercambio con el otro y una construcción dialógica (Sisto, 2008). Investigadores e investigados debemos darnos la posibilidad de explicitar nuestros marcos de referencia académicos y existenciales; expresar nuestras ideas, creencias y convicciones; decir la propia palabra; valorar nuestras formas de vida y respetar las de los demás; ser escuchados y poder escuchar al otro, sin perder de vista que, como hemos observado en nuestras investigaciones, la participación no elimina las relaciones de poder, sino que busca una alternativa democrática de las mismas.

La participación precisa un ejercicio de construcción colaborativa, sostenido y realizado con madurez, responsabilidad y con el compromiso de todos los actores involucrados, aunque el esfuerzo recaiga, sobre todo, en el investigador. Entendemos que la validez del conocimiento que se construye desde perspectivas interpretativas y críticas depende, en última instancia, del rigor y de la integridad de los investigadores.

Esto es así aun en prácticas de investigación-acción participante. Quienes coordinan el trabajo, los docentes investigadores más interesados, los más comprometidos con los propósitos de la investigación y los que tienen mayor capacidad para escribir son las personas que más intervienen en la toma de decisiones, en el control de las acciones acordadas y, en definitiva, ejercen una mayor cuota de poder en el proceso de construcción del conocimiento. Hay voces, en cambio, que quedan olvidadas, silenciadas y relegadas. Como hemos evidenciado en este artículo, en estos actores hay historias, saberes, temores, lealtades y alianzas, que los posicionan en inferioridad de condiciones a la hora de participar.

Dado que formamos parte del mundo que investigamos, que sustentamos visiones de mundo diferentes y que la distribución diferencial del poder es inevitable entre investigadores e investigados, el conocimiento que construimos implica evaluación y juicio, tanto en los estudios etnográficos como en las comunidades autocríticas de investigación educativa. Como dice Schuster (2002):

Habermas va mucho más allá de decir que la neutralidad valorativa es imposible: por un lado, este autor afirma que la neutralidad valorativa es ideológica, falsa, y, por el otro, que el juicio es una condición necesaria para poder explicar en Ciencias Sociales (p. 239). 
En tal sentido, las perspectivas emancipadoras postulan la crítica ideológica como herramienta intelectual para develar las fuerzas y los intereses que intervienen en las complejas tramas de poder. En esta línea, sostenemos que la necesidad de desenmascarar la ideología del discurso pedagógico dominante no solo justifica, sino que exige un conocimiento que ligue condiciones macroestructurales con la intención de los actores y la búsqueda de propuestas educativas alternativas.

Para concluir, queremos agradecer a quienes nos han dado la posibilidad de publicar estas reflexiones sobre los dilemas éticos que enfrentamos en nuestras prácticas de investigación educativa y dejar planteada la necesidad de introducirlas en los procesos de enseñanza y aprendizaje.

\section{Sobre las autoras}

Lorena Cruz-Garcette es doctora por la Universidad de Barcelona, becaria posdoctoral del Conicet, Argentina. Es investigadora del Instituto de Ciencias de la Educación, de la Facultad de Filosofía y Letras, de la Universidad Nacional de Cuyo, Argentina. Miembro externo del grupo de investigación FODIP-DOE, de la Universitat de Barcelona, España. Estancia de investigación Universidad de Utrecht, Holanda.

Graciela Martins de Abreu-Ballester es docente investigadora y asesora técnica curricular en el nivel superior universitario y miembro de la Comisión Directiva del Instituto de Ciencias de la Educación.

Lía Stella Brandi-Bruna es magíster en Investigación Educativa. Se desempeña como directora del Instituto de Ciencias de la Educación, de la Facultad de Filosofía y Letras, de la Universidad Nacional de Cuyo. Ha sido profesora titular en la Universidad Nacional de Cuyo y en la Universidad Nacional de San Juan y ha dirigido diversas investigaciones educativas de enfoque cualitativo.

\section{Referencias}

Ben-Ari, A., \& Enosh, G. (2010). Processes of Reflectivity: Knowledge Construction in Qualitative Research. Qualitative Social Work, 10(2), 152-171.

Ben-Ari, A., \& Enosh, G. (2013). Power Relations and Reciprocity: Dialectics of Knowledge Construction. Qualitative Health Research, 23(3), 422-429.

Bertely-Busquets, M. (2000). Conociendo nuestras escuelas. Un acercamiento etnográfico a la cultura escolar. México D. F.: Paidós

Braslavsky, C. (1985). La discriminación educativa en la Argentina. Buenos Aires: GEL- Flacso.

Breuer, F. (2003). Subjectivity and Reflexivity in the Social Sciences: Epistemic Windows and Methodical Consequences. Forum: Qualitative Social
Research, 4(2). Recuperado de http://nbn-resolving.de/urn:nbn:de:0114-fqs0302258

Carr, W., \& Kemmis, S. (1988). Teoría crítica de la enseñanza: la investigación-acción en la formación del profesorado. Barcelona: Martínez Roca.

Chevallard, Y. (1991). La transposición didáctica. Del saber sabio al saber enseñado. (3a ed.) Buenos Aires: Aique.

Cornejo, M., Besoaín, C., \& Mendoza, F. (2011). Desafíos en la generación de conocimiento en la investigación social cualitativa contemporánea. Forum: Qualitative Social Research, 12(1). Recuperado de http://www.qualitative-research.net/ index.php/fqs/article/view/1527/3140

Cornejo, M., \& Salas, N. (2012). Rigor y calidad metodológicos: un reto a la investigación social cualitativa. Psicoperspectivas, 10(2), 12-34.

Declan, F. (2014). Doing Research Sensitively: Ethical and Methodological Issues in Researching Workplace Bullying. International Journal of Qualitative Methods, 13, 13-36.

Enosh, G., \& Ben-Ari, A. (2009). Cooperation and Conflict in Qualitative Research: A Dialectical Approach to Knowledge Production. Qualitative Health Research, 20(1), 125-130.

Foucault, M. (1999). Estética, ética y hermenéutica. (A. Gabilondo, trad.). Barcelona: Paidós. (Original publicado en 1994).

Giroux, H., \& Simon, R. (1998). Pedagogía crítica y políticas de cultura popular. En H. Giroux, \& P. Mc Laren. (eds.). Sociedad, cultura y educación. Madrid: Miño y Dávila editores.

Goffman, E. (1974). Les rites $d^{\prime}$ interaction. París: Minuit.

Habermas, J. (1982). Conocimiento e interés. Madrid: Taurus.

Karnieli-Miller, O., Strier, R., \& Pessach, L. (2009). Power Relations in Qualitative Research. Qualitative Health Research, 19(2), 279-289.

Kuhn, T. (2006). La estructura de las revoluciones científicas. 3a ed. México D. F.: Fondo de Cultura Económica.

Lim, M. (2012). Being a Korean Studying Koreans in an American School: Reflections on Culture, Power and Ideology. The Qualitative Report, 17, 1-15. Recuperado de http://www.nova.edu/sss/QR/ QR17/lim.pdf

Pérez-Gómez, Á. (1993). La función y formación del profesor/a en la enseñanza para la comprensión. Diferentes perspectivas. En Gimeno-Sacristán, J., \& Pérez-Gómez, A. (eds.). Comprender y transformar la enseñanza. (2a ed.). Madrid: Morata.

Schuster, F. (2002). Filosofía y métodos de las Ciencias Sociales. Buenos Aires: Manantial. 
Schuster, F., \& Pecheny, M. (2002). Objetividad sin neutralidad valorativa según Jürgen Habermas. En Schuster, F. (ed.). Filosofía y métodos de las Ciencias Sociales. Buenos Aires: Manantial.

Sirvent, M. T. (1998). El proceso de investigación y las dimensiones de la metodología y la construcción del dato científico. Documento de cátedra, Universidad de Buenos Aires, Argentina.

Sisto, V. (2008). La investigación como una aventura de producción dialógica: la relación con el otro y los criterios de validación en la metodología cualitativa contemporánea. Psicoperspectivas, VII, 114-136.

Vasilachis, I. (1992). Métodos cualitativos I. Los problemas teórico-epistemológicos. Buenos Aires: Centro Editor de América Latina.

Vasilachis, I. (2009). Los fundamentos ontológicos y epistemológicos de la investigación cualitativa. Forum: Qualitative Social Research, 10(2). Recuperado de http://www.qualitative-research.net/index.php/fqs/ article/view/1299

Vorraber, M. (1997). Elementos para una crítica de las metodologías participativas de investigación. En Veiga-Neto, A. (ed.). Crítica pos-estructuralista y educación. Barcelona: Laertes.

Wiesenfeld, E. (2000). Entre la prescripción y la acción: la brecha entre la teoría y la práctica en las investigaciones cualitativas. Forum: Qualitative Social Research, 1(2). Recuperado de http://www.qualitativeresearch.net/index.php/fqs/article/view/1099/2419

Willis, P. (1980). Notas sobre el método. Santiago de Chile: Red latinoamericana de investigaciones cualitativas de la realidad escolar. 